\title{
Mapeamento dos estudos transversais no PUBMED sobre a associação entre Síndrome de Stevens-Johnson e cegueira: estudo de prevalência
}

\author{
Mapping of cross-sectional studies at PUBMED on the association between Stevens- \\ Johnson Syndrome and blindness: prevalence study
}

Mapeo de estudios transversales en PUBMED sobre la asociación entre el síndrome de Stevens-Johnson y la ceguera: estudio de prevalencia

Thales José Moura Moraes $^{1 *}$, Felipe Monteiro Carvalheiro ${ }^{1}$, Renan Monteiro da Silveira ${ }^{1}$, Brenda Nazaré Gomes Andriolo'.

\section{RESUMO}

Objetivo: Analisar estudos que avaliam a associação entre Síndrome de Stevens-Johnson e cegueira disponíveis no PUBMED, identificar as principais publicações a respeito do assunto e conhecer as taxas de associação. Métodos: Trata-se de estudo transversal analítico, conduzido com 7 artigos observacionais e transversais, selecionados independentemente do idioma. Os artigos selecionados foram classificados e avaliados, com base nos critérios propostos pela iniciativa STROBE (Strengthening the Reporting of Observational Studies in Epidemiology), que contém itens com recomendações do que é necessário conter em um estudo observacional de qualidade. Para cada capítulo julgado, os artigos foram classificados como: adequado; inadequado e insuficiente. Resultados: Dos tópicos preconizados pela iniciativa STROBE, "Título e Resumo" foi o que ficou com maior percentual em adequado, 92,85\%, enquanto "Outras Informações", onde os autores discorrem sobre o financiamento da pesquisa, ficou com a maior porcentagem em inadequado, $100 \%$ e "Introdução" o maior percentual em insuficiente, $28,57 \%$. Quanto às taxas de associação, essas ficaram entre $2,2 \%$ e $60 \%$. Considerações finais: Conclui-se que há uma dificuldade para os pesquisadores em organizar e expor seu financiamento e que as taxas de associação são heterogêneas, dependendo da metodologia proposta.

Palavras-chave: Síndrome de Stevens-Johnson, Cegueira, Prevalência.

\section{ABSTRACT}

Objective: To analyze studies that evaluate the association between Stevens-Johnson Syndrome and blindness available at PUBMED, to identify the main publications on the subject and to know the association rates. Methods: This is a cross-sectional analytical study, conducted with 7 observational and cross-sectional articles, selected regardless of language. The selected articles were classified and evaluated, based on the criteria proposed by the STROBE (Strengthening the Reporting of Observational Studies in Epidemiology) initiative, which contains items with recommendations of what is necessary to contain in a quality observational study. For each chapter judged, the articles were classified as: adequate; inadequate and insufficient. Results: Of the topics recommended by the STROBE initiative, "Title and Summary" was the one with the highest percentage in appropriate, $92.85 \%$, while "Other Information", where the authors write about research funding, got the highest percentage in inadequate, $100 \%$ and "Introduction" the highest percentage in insufficient, $28.57 \%$. As for association fees, these were between $2.2 \%$ and $60 \%$. Final considerations: It is concluded that there is a difficulty for researchers to organize and expose their funding and that the association fees are heterogeneous, depending on the proposed methodology.

Keywords: Stevens-Johnson Syndrome, Blindness, Epidemiology.

\section{RESUMEN}

Objetivo: Analizar estudios que evalúen la asociación entre el síndrome de Stevens-Johnson y la ceguera disponible en PUBMED, identificar las principales publicaciones sobre el tema y conocer las tasas de asociación. Métodos: Este es un estudio analítico transversal, realizado con 7 artículos observacionales y transversales, seleccionados independientemente del idioma. Los artículos seleccionados se clasificaron y evaluaron, de acuerdo con los criterios propuestos por la iniciativa STROBE (Strengthening the Reporting of

1 Universidade do Estado do Pará (UEPA), Belém - PA. *E-mail: thales_doc@hotmail.com 
Observational Studies in Epidemiology) que contiene elementos con recomendaciones de lo que es necesario contener en un estudio observacional de calidad. Para cada capítulo juzgado, los artículos fueron clasificados como: adecuados; inadecuado e insuficiente. Resultados: De los temas recomendados por la iniciativa STROBE, "Título y resumen" fue el que tuvo el porcentaje más alto en el apropiado, $92.85 \%$, mientras que "Otra información", donde los autores hablan sobre la financiación de la investigación, obtuvo el porcentaje más alto en inadecuado, $100 \%$ e "Introducción" el porcentaje más alto en insuficiente, $28.57 \%$. En cuanto a las cuotas de membresía, estas fueron entre $2.2 \%$ y $60 \%$. Consideraciones finales: Se concluye que existe una dificultad para los investigadores para organizar y exponer sus fondos y que las tarifas de asociación son heterogéneas, dependiendo de la metodología propuesta.

Palabras clave: Síndrome de Stevens-Johnson, Ceguera, Epidemiología.

\section{INTRODUÇÃO}

A Síndrome de Stevens-Johnson (SSJ) é uma patologia mucocutânea aguda, pouco comum e potencialmente fatal que apresenta como principal característica a reação de pele, resultante da necrose de queratinócitos, expressada clinicamente por descolamento epidérmico (WONG A, et al., 2016).

A SSJ é habitualmente enquadrada nas reações cutâneas adversas graves a fármacos de utilização sistêmica, embora alguns casos sejam atribuídos a outras causas como infecções (BRINCA A, et al., 2011).

Em 1922, Stevens e Johnson iniciaram o relato do que seria um novo grupo dessas reações e, após futuros estudos, Bastuji-Garin S, et al. (1993), propuseram a classificação usada atualmente, na qual são definidos o Eritema Polimorfo Bolhoso, a SSJ e a Necrólise Epidérmica Tóxica (NET), o primeiro como uma entidade patológica diferente dos dois últimos, já a SSJ e a NET como manifestações do mesmo espectro patológico, diferenciando-se pela gravidade (OLIVEIRA A, et al., 2011).

A NET representa uma forma mais severa da doença, com um descolamento epidérmico superior a $30 \%$ da superfície corporal, enquanto que na SSJ esse valor não passa de $10 \%$, com aumento da área acometida representando um proporcional mau prognóstico (RUIZ RB, et al., 2019).

Sua incidência no mundo é de 1,2-6 casos/milhão de habitante para a forma menos agressiva e de 0,41,2 caso/milhão de habitante para a mais grave. A mortalidade do espectro da SSJ varia conforme a gravidade do acometimento mucocutâneo podendo ir de 1 a $5 \%$ na sua forma mais branda, até $35 \%$ na sua forma mais severa (BRINCA A, et al., 2011; OLIVEIRA A, et al., 2011; RUIZ RB, et al., 2019).

Diversos são os fatores de risco documentados, como: gênero feminino, idade elevada, predisposição genética, imunossupressão iatrogênica, neoplasias malignas e infecção pelo Vírus da Imunodeficiência Humana (HIV) (OSHIKOYA K, et al., 2020).

O banco de dados a respeito desta síndrome e suas complicações é bastante escasso fora da Europa, onde se iniciou a consolidação das publicações sobre SSJ na década de 90, especialmente em países como França e Alemanha (MOCKENHAUPT M, 2011).

A SSJ pode afetar vários outros órgãos, de forma aguda e/ou crônica, na forma de sequelas, como rins, fígado, pulmões e olhos (DAY A, et al., 2014). Complicações oculares nessa síndrome podem ocorrer de forma aguda junto ou após o envolvimento cutâneo em 50-67\% dos casos, além disso, essas complicações podem resultar em permanente perda visual em decorrência do processo cicatricial da córnea e da vascularização (TCHETNYA X, et al., 2018).

Como podemos observar nos estudos de Estrella-Afonso A, et al. (2017) e Ueta M (2018), quadro oftalmológico agudo é caracterizado por uma conjuntivite catarral e purulenta bilateral, com defeitos epiteliais na superfície ocular (córnea e/ou conjuntiva). Apesar de rara, a síndrome gera um forte impacto social e econômico, já que se trata de uma patologia com características crônicas que leva, potencialmente, à cegueira pacientes numa faixa etária, em muitos casos, produtiva (SCHWERING MS, et al., 2019).

A cronicidade das complicações da SSJ e os desafios para o acompanhamento dos pacientes são preocupações para a saúde pública, pois se trata de uma condição de grande impacto funcional sobre a vida das pessoas, trazendo mudanças bruscas e novos desafios na sociedade (CHRONPOULOS A, et al., 2012). 
A cegueira atinge mais de 14 milhões de crianças no globo, e está fortemente ligada a países subdesenvolvidos e com altas taxas de mortalidade nos primeiros anos de vida em decorrência de doenças associadas ao comprometimento visual (JUNIOR AC e OLIVEIRA LAG, 2016; SOLEBO A, et al., 2017).

Vale destacar que mesmo que a cegueira seja apontada como um efeito da Síndrome de StevensJohnson, ainda poucos os trabalhos disponíveis que abordam a associação entre elas. Portanto, este trabalho justifica-se por compilar estudos que abordam a temática, a fim de produzir o conhecimento e a compreensão da produção científica existente entre a associação da Síndrome de Stevens-Johnson e a cegueira.

Além disso, o estudo desses dados pode proporcionar um melhor entendimento a respeito da etiologia, dos fatores de risco, do acompanhamento e do tratamento, possibilitando o desenvolvimento de protocolos mais modernos, consolidados para atender e melhorar a saúde ocular dos pacientes e estudos epidemiológicos fornecem importantes insights sobre a extensão do problema (BRINCA A, et al., 2011; UETA $\mathrm{M}, 2018)$.

Dessa forma, em linhas gerais, a fim de contribuir científica e epidemiologicamente, o presente estudo tem como objetivos: 1 - analisar artigos disponíveis no PUBMED Central (PMC), que avaliam a associação de Síndrome de Stevens-Johnson com a cegueira, por meio de um checklist conhecido como iniciativa STROBE (Strengthening the Reporting of Observational Studies in Epidemiology), que reúne um conjunto de recomendações sobre que tipo de informações que devem constar nos artigos; 2- Identificar as principais publicações à respeito à associação de Síndrome de Stevens-Johnson com a cegueira e conhecer as taxas de associação.

\section{MÉTODOS}

O presente estudo caracterizou-se como uma pesquisa transversal analítica. Foi realizado um mecanismo de busca sensível para realizar a pesquisa por artigos sobre a associação da Síndrome de Steven-Johnson e cegueira, que combinou termos oficiais e sinônimos desta temática. As buscas foram realizadas por meio dos seguintes descritores: ("Stevens-Johnson Syndrome"[Mesh]) OR (Stevens Johnson Syndrome) OR (Stevens-Johnson Syndrome Toxic Epidermal Necrolysis Spectrum) OR (Stevens Johnson Syndrome Toxic Epidermal Necrolysis Spectrum) OR (Toxic Epidermal Necrolysis Stevens-Johnson Syndrome Spectrum) OR (Toxic Epidermal Necrolysis Stevens Johnson Syndrome) OR (Toxic Epidermal Necrolysis Stevens Johnson Syndrome Spectrum) OR (Toxic Epidermal Necrolysis Stevens-Johnson Syndrome) OR (Stevens Johnson Syndrome Toxic Epidermal Necrolysis) OR (Stevens-Johnson (Syndrome Toxic Epidermal Necrolysis) OR (Mycoplasma-Induced Stevens-(Johnson Syndrome) OR (Mycoplasma Induced Stevens Johnson Syndrome) OR (Stevens-Johnson Syndrome, Mycoplasma-Induced) OR (Syndrome, Mycoplasma-Induced StevensJohnson) OR (Mycoplasma-Induced Stevens Johnson Syndrome) OR (Drug-Induced Stevens Johnson Syndrome) OR (Drug Induced Stevens Johnson Syndrome) OR (Drug-Induced Stevens-Johnson Syndrome) OR (Drug-Induced Stevens-Johnson Syndromes) OR (Stevens-Johnson Syndrome, Drug-Induced) OR (Stevens-Johnson Syndromes, Drug-Induced) OR (Epidermal Necrolysis, Toxic) OR (Epidermal Necrolyses, Toxic) OR (Necrolyses, Toxic Epidermal) OR (Necrolysis, Toxic Epidermal) OR (Toxic (Epidermal Necrolyses) OR (Toxic Epidermal Necrolysis) OR (Scalded Skin (Syndrome, Nonstaphylococcal) OR (Lyell's Syndrome) OR (Lyell Syndrome) OR (Lyell's Syndromes) OR (Syndrome, Lyell's) OR (Syndromes, Lyell's) OR (Nonstaphylococcal Scalded Skin Syndrome) AND ("Blindness"[Mesh]) OR (Blindness Bilateral) OR (Blindness, Bilateral) OR (Bilateral Blindness) OR (Bilateral Blindnesses) OR (Amaurosis) OR (Amauroses) OR (Blindness, Acquired) OR (Acquired Blindness) OR (Sudden Visual Loss) OR (Sudden Visual Losses) OR (Visual Loss, Sudden) OR (Visual Loss Sudden) OR (Sudden, Visual Loss) OR (Visual Loss Suddens) OR (Blindness, Hysterical) OR (Hysterical Blindness) OR (Blindness, Transient) OR (Transient Blindness) OR (Blindness Transient) OR (Transient, Blindness) OR (Blindness, Legal) OR (Legal Blindness) OR (Blindness, Monocular) OR (Monocular Blindness) OR (Blindness, Unilateral) OR (Unilateral Blindness) OR (Blindness Unilateral) OR (Unilateral, Blindness) OR (Blindness, Complete) OR (Complete Blindness).

As buscas foram realizadas desde o início da criação da base de dados em setembro de 2019 até janeiro de 2020. Os estudos foram selecionados para compor a amostra, a partir da leitura dos títulos e resumos, de 
forma independente. Não foram estabelecidos limites para o ano de publicação, nem distinção quanto à língua da publicação. Foram selecionados artigos publicados, independentemente da língua, observacionais e transversais, que avaliaram qualquer associação entre Síndrome de Stevens-Johnson e cegueira, localizados na base de dados PUBMED. Assim como foram excluídos os estudos cujos desenhos não se caracterizavam em observacionais e transversais; não apresentavam associação entre Síndrome de Stevens- Johnson e cegueira.

Nove artigos se encaixaram nos critérios propostos, porém os autores só tiveram acesso a sete, os quais entraram para a pesquisa. Os artigos selecionados passaram por uma avaliação de qualidade, de acordo com as recomendações da iniciativa STROBE, que constitui em um conjunto de recomendações para melhorar a qualidade dos relatórios de estudos observacionais (VANDENBROUCKE JP, et al., 2014).

Desta forma, foram avaliados 6 tópicos referentes aos capítulos dos projetos (título/resumo, introdução, método, resultados, discussão, outras informações), totalizando 23 subtópicos (especificados na tabela 2), que, após a leitura de cada estudo foram, classificados em: 1) Adequado - se preencheu todos, senão a maioria, dos pré-requisitos da iniciativa STROBE para aquele capítulo; 2) Inadequado - se não preencheu os pré-requisitos estabelecidos pela iniciativa STROBE para aquele capítulo; 3) Insuficiente - se o artigo não apresentou informações suficientes para análise adequada daquele capítulo, de acordo com a iniciativa STROBE.

Os dados colhidos em cada artigo foram tabulados e analisados em planilhas digitais. A presente pesquisa analisou estudos publicados, com aprovação prévia do comitê de ética, e por esta razão, não precisa de nova submissão, uma vez que nenhum indivíduo sofreu qualquer intervenção.

\section{RESULTADOS}

Os estudos foram desenvolvidos nos Estados Unidos da América - EUA, Japão, França, Brasil, África do Sul e Índia (SHANBHAG SS, et al., 2019; YAMANE Y, et al., 2007; GUEUDRY J, et al., 2009; FRANCA M, et al., 2009; VAN ZYL L, et al., 2014; BASU S, et al., 2018; KOMPELLA VB, et al., 2002). Quanta às taxas de associação entre SSJ e cegueira encontradas, o estudo de Basu S, et al. (2018) foi o que apresentou o maior percentual, $60 \%$, sendo também o com maior tamanho amostral. Já a menor taxa foi encontrada no estudo de Gueudry J, et al. (2009), de 2,2\% (Tabela 1).

Tabela 1 - Caracterização dos artigos analisados.

\begin{tabular}{|c|c|c|c|c|c|}
\hline Autor & País & Amostra & População-alvo & $\begin{array}{c}\text { Olhos } \\
\text { acometidos por } \\
\text { cegueira }\end{array}$ & Associação \\
\hline $\begin{array}{l}\text { Kompella VB, et al. } \\
(2002)\end{array}$ & Índia & $\begin{array}{c}95 \\
\text { (190 olhos) }\end{array}$ & Geral & 8 & $4,21 \%$ \\
\hline $\begin{array}{l}\text { Yamane Y, et al. } \\
(2007)\end{array}$ & Japão & $\begin{array}{c}117 \\
\text { (234 olhos) }\end{array}$ & Geral & 16 & $6,83 \%$ \\
\hline $\begin{array}{c}\text { Gueudry J, et al. } \\
(2009)\end{array}$ & França & $\begin{array}{c}159 \\
\text { (318 olhos) }\end{array}$ & Geral & 7 & $2,20 \%$ \\
\hline $\begin{array}{l}\text { Franca M, et al. } \\
\qquad(2009)\end{array}$ & Brasil & $\begin{array}{c}22 \\
\text { (44 olhos) }\end{array}$ & Geral & 2 & $4,54 \%$ \\
\hline $\begin{array}{c}\text { Van Zyl L, et al. } \\
(2014)\end{array}$ & $\begin{array}{l}\text { África do } \\
\text { Sul }\end{array}$ & $\begin{array}{c}54 \\
\text { (108 olhos) }\end{array}$ & Geral & 7 & $6,48 \%$ \\
\hline $\begin{array}{l}\text { Basu S, et al. } \\
\quad(2018)\end{array}$ & Índia & $\begin{array}{c}284 \\
\text { (568 olhos) }\end{array}$ & $\begin{array}{c}\text { Crianças ( }<16 \text { anos } \\
\text { ou }=\text { a } 16 \text { anos) }\end{array}$ & 338 & $60 \%$ \\
\hline $\begin{array}{l}\text { Shanbhag SS, et } \\
\text { al. (2019) }\end{array}$ & EUA & $\begin{array}{c}48 \\
\text { (96 olhos) }\end{array}$ & Geral & 16 & $16,66 \%$ \\
\hline
\end{tabular}

Fonte: Moraes TJM, et al., 2020.

Podemos observar que os subtópicos "Resumo informativo e equilibrado", "Desenho do estudo", "Tamanho do estudo", "Participantes", "Outras análises", "Resultados principais" e "Interpretação" se apresentaram 
adequados em todos os estudos. Já os subtópicos "Viés" e "Financiamento" estiveram inadequados em todos os estudos (Tabela 2).

Tabela 2 - Classificação dos 23 subtópicos categorizados segundo iniciativa STROBE nos artigos.

\begin{tabular}{cccc}
\hline Subtópicos STROBE/Classificação & Adequado & Inadequado & Insuficiente \\
\hline 1a - Desenho do estudo no título e/ou resumo & $6(85,71 \%)$ & $1(14,28 \%)$ & $0(0,00 \%)$ \\
1b- Resumo informativo e equilibrado & $7(100,00 \%)$ & $0(0,00 \%)$ & $0(0,00 \%)$ \\
2- Contexto/ Justificativa (Introdução) & $6(85,71 \%)$ & $0(0,00 \%)$ & $1(14,28 \%)$ \\
3- Objetivos (Introdução) & $4(57,14 \%)$ & $0(0,00 \%)$ & $3(42,85 \%)$ \\
4- Desenho do estudo (Método) & $7(100,00 \%)$ & $0(0,00 \%)$ & $0(0,00 \%)$ \\
5- Contexto (Método) & $5(71,42 \%)$ & $1(14,28 \%)$ & $1(14,28 \%)$ \\
6- Participantes (Método) & $6(85,71 \%)$ & $0(0,00 \%)$ & $1(14,28 \%)$ \\
7- Variáveis (Método) & $1(14,28 \%)$ & $1(14,28 \%)$ & $5(71,42 \%)$ \\
9- Vontes (Método) & $3(42,85 \%)$ & $1(14,28 \%)$ & $3(42,85 \%)$ \\
10- Tamanho do estudo (Método) & $0(0,00 \%)$ & $7(100,00 \%)$ & $0(0,00 \%)$ \\
11- Variáveis quantitativas (Método) & $7(100,00 \%)$ & $0(0,00 \%)$ & $0(0,00 \%)$ \\
12- Métodos estatísticos (Método) & $4(57,14 \%)$ & $0(0,00 \%)$ & $3(42,85 \%)$ \\
13- Participantes (Resultados) & $1(14,28 \%)$ & $3(42,85 \%)$ & $3(42,85 \%)$ \\
14- Dados descritivos (Resultados) & $7(100,00 \%)$ & $0(0,00 \%)$ & $0(0,00 \%)$ \\
15- Desfecho (Resultados) & $2(28,57 \%)$ & $2(28,57 \%)$ & $3(42,85 \%)$ \\
16- Resultados principais (Resultados) & $5(71,42 \%)$ & $1(14,28 \%)$ & $1(14,28 \%)$ \\
17- Outras análises (Resultados) & $2(28,57 \%)$ & $3(42,85 \%)$ & $2(28,57 \%)$ \\
18- Resultados principais (Discussão) & $7(100,00 \%)$ & $0(0,00 \%)$ & $0(0,00 \%)$ \\
19- Limitações (Discussão) & $7(100,00 \%)$ & $0(0,00 \%)$ & $0(0,00 \%)$ \\
20- Interpretação (Discussão) & $4(57,14 \%)$ & $3(42,85 \%)$ & $0(0,00 \%)$ \\
21- Generalização (Discussão) & $7(100,00 \%)$ & $0(0,00 \%)$ & $0(0,00 \%)$ \\
22- - Financiamento (Outras informações) & $5(71,42 \%)$ & $0(0,00 \%)$ & $2(28,57 \%)$ \\
\hline Total & $0(0,00 \%)$ & $7(100,00 \%)$ & $0(0,00 \%)$ \\
\hline
\end{tabular}

Fonte: Moraes TJM, et al., 2020.

Analisando os tópicos propostos pela iniciativa, é possível identificar que "Título e resumo" foi o tópico com melhor desempenho entre os artigos, atingindo um percentual de $92,85 \%$ de adequado, ficando inadequado em apenas $7,14 \%$. Ainda avaliando os tópicos, "Outras informações" foi o que recebeu a maior porcentagem em inadequado, atingindo $100 \%$ das publicações (Tabela 3).

Tabela 3 - Classificação dos 6 tópicos categorizados segundo iniciativa STROBE nos artigos.

\begin{tabular}{ccccccc}
\hline $\begin{array}{c}\text { Classificação/Tópico } \\
\text { STROBE }\end{array}$ & $\begin{array}{c}\text { Título e } \\
\text { Resumo }\end{array}$ & Introdução & Método & Resultados & Discussão & $\begin{array}{c}\text { Outras } \\
\text { Informações }\end{array}$ \\
\hline Adequado & 13 & 10 & 34 & 23 & 23 & 0 \\
& $(92,85 \%)$ & $(71,42 \%)$ & $(53,96 \%)$ & $(67,64 \%)$ & $(82,14 \%)$ & $(0,00 \%)$ \\
Inadequado & 1 & 0 & 13 & 6 & 3 & 7 \\
& $(7,14 \%)$ & $(0,00 \%)$ & $(20,63 \%)$ & $(17,64 \%)$ & $(10,71 \%)$ & $(100,00 \%)$ \\
Insuficiente & 0 & 4 & 16 & 5 & 2 & 0 \\
& $(0,00 \%)$ & $(28,57 \%)$ & $(25,39 \%)$ & $(14,70 \%)$ & $(7,14 \%)$ & $(0,00 \%)$ \\
\hline \multirow{2}{*}{ Total } & 14 & 14 & 63 & 34 & 28 & 7 \\
& $(100,00 \%)$ & $(100,00 \%)$ & $(100,00 \%)$ & $(100,00 \%)$ & $(100,00 \%)$ & $(100,00 \%)$ \\
\hline
\end{tabular}

Fonte: Moraes TJM, et al., 2020. 


\section{DISCUSSÃO}

Até onde se tem ciência o presente trabalho constitui o primeiro mapeamento realizado analisando os estudos que associam a Síndrome de Stevens- Johnson e cegueira. Este mapeamento proporciona um olhar panorâmico acerca dos distúrbios abordados e as pesquisas realizadas sobre o assunto, além de permitir uma categorização qualitativa destas com uma abordagem segundo a iniciativa STROBE.

De acordo com o objetivo primário deste trabalho, que foi analisar os artigos publicados na base de dados PUBMED que fizessem associação entre SSJ e cegueira, foi constatada grande diversidade entre estes, tanto qualitativamente quanto ao que se propunham a estudar. As publicações são de vários países e continentes, sendo apenas duas do mesmo país. Porém, devido às dificuldades para a realização das pesquisas com SSJ, sua raridade e uma taxa de incidência de cegueira em pacientes com SSJ não consolidada, houve uma grande dificuldade para encontrar estudos que fizessem essa associação, resultando em um baixo número amostral, mesmo sem limitar pelo idioma da publicação.

As associações entre SSJ e cegueira encontradas nos trabalhos analisados, e destacadas na Tabela 1, mostraram-se bastante heterogêneas, fato este que se deve não só a falta de padronização entre os diversos estudos quanto ao que de fato analisavam, como também ao tamanho amostral tão diversificado entre os trabalhos. Essa diferença amostral é bastante significativa, variando entre 22 indivíduos, no trabalho de Franca M, et al. (2009), a 284 indivíduos, no estudo de Basu S, et al. (2018).

O estudo que apresentou a maior taxa de associação é também o maior, o de Basu $S$ et al. (2018), porém os dados apresentados pelo autor podem não corresponder à real associação com a cegueira, visto que o autor seleciona os pacientes com perda visual e cegueira para o mesmo grupo. Tal escolha faz a taxa de associação não ser confiável, pois a perda visual é comum entre os pacientes com acometimento oftalmológico na SSJ, sendo relatada amplamente por todos os artigos. Assim, pode-se concluir que esse número está, possivelmente, inflado.

Já o estudo com a segunda maior taxa de associação foi o de Shanbhag SS, et al. (2019) e a segunda menor amostra. Assim, a heterogeneidade dos resultados nos faz questionar o papel de cada componente desses números, condições patológicas, socioeconômicas, amostra, critérios de seleção e metodologia. É importante a análise do perfil epidemiológico dos estudos, já que as faixas etárias são muito variadas, o estudo de Basu S, et al. (2018), por exemplo, só estudou crianças, enquanto os outros não limitaram por idade, já o de Van Zyl L, et al. (2014) foi o único que incluiu pacientes portadores de HIV e comparou. Outro fator importante é o tempo de acompanhamento de cada estudo, bastante variado entre eles.

Também se verificou que os estudos também tiveram diferentes fontes de dados, estudos como os de Basu S, et al. (2018), Franca M, et al. (2009), Gueudry J, et al. (2009) e Kompella VB, et al. (2002) utilizaram as informações disponíveis de 1 único serviço terciário de oftalmologia, já Shanbhag SS, et al. (2019) lançou mão de 2 hospitais de referência em problemas oculares nos EUA. Yamane Y, et al. (2007) analisaram a partir dos dados de todo o país e Van Zyl L, et al. (2014) coletaram os dados a partir de um centro terciário de oftalmologia.

Vale salientar que a falta de qualidade metodológica avaliada de acordo com a iniciativa STROBE também pode ser fator determinante para a divergência encontrada entre os trabalhos analisados, uma vez que diversos dados foram omitidos e/ou não devidamente coletados, impossibilitando a devida comparação.

É preciso conhecer e compreender as diversas dificuldades que os pesquisadores encontram ao estudar as complicações oftalmológicas da SSJ, como a escassa base de dados referente à doença fora da Europa, a raridade da doença, a demora para os casos chegarem ao oftalmologista e o longo tempo necessário para o acompanhamento (FRANCA M, et al., 2009; GUEUDRY J, et al., 2009; HARR T e FRENCH L, 2010; KOMPELLA VB, et al., 2002; SHANBHAG SS, et al., 2019).

A raridade da doença, que dificulta muito o estabelecimento de uma amostra robusta. A incidência varia em torno de 1,2 casos por milhão de habitantes, quando é dado o enfoque nas complicações oculares, esse número ainda cai bastante e este fato é apontado por todos os pesquisadores como principal motivo para os 
números baixos de amostra. No estudo de Kompella VB et al. (2002), realizado num centro de referência oftalmológica na Índia, a prevalência no centro chegou a 0,0004\% (BRINCA A, et al., 2011; OLIVEIRA A, et al., 2011; WONG A, et al., 2016).

Além disso, a demora para o paciente chegar ao centro especializado de oftalmologia e o longo período de acompanhamento que a SSJ demanda são grandes problemas a serem enfrentados. Essa demora se dá devido ao quadro agudo ser, em grande parte dos casos, dermatológico, assim, o paciente não é avaliado por oftalmologista, nem referenciado após a resolução do quadro cutâneo. As queixas do paciente podem ser subestimadas ou nem avaliadas até que já haja prejuízo visual e, mesmo os pacientes que não apresentam queixas oculares na fase aguda podem vir a desenvolver complicação oftalmológica (ESTRELLA-AFONSO A, et al., 2018; HARR T e FRENCH L, 2010; UETA M, 2018).

Essa questão corrobora para compor o cenário atual de subnotificação e dificuldades que os sistemas de saúdem têm para atender o paciente com SSJ de maneira global e multiprofissional (IYER G, et al., 2016; SCHWERING MS, et al., 2019).

O tópico da iniciativa STROBE que se mostrou mais adequado na maioria dos estudos analisados foi o "Título/Resumo", fato este que pode ser atribuído a pouca complexidade exigida para tal e/ou ao fato deste tópico possuir mais de um subtópico.

Por outro lado, os tópicos "Método" e "Outras Informações" foram os que figuraram com maior inadequação, o que retrata a dificuldade que a maioria dos autores têm em fundamentar suas pesquisas nestes que são pontos fundamentais para o bom entendimento e relevância do assunto perante a comunidade cientifica, e dificuldade para expor de maneira clara e detalhada o que irá ser estudado, como irá ser estudado e os resultados desse estudo.

"Viés" foi o subtópico do tópico "Método" que mais se mostrou inadequado, se apresentando assim em todos os artigos, demostrando a dificuldade existente em conseguir se determinar com precisão os vieses, expô-los e discuti-los ou ainda transmitir com clareza o que desejava. Em relação ao tópico "Outras Informações", esse foi o tópico com maior taxa de inadequação (100\%), nenhum dos artigos detalhou seu financiamento. A taxa ficou tão alta devido ao tópico só se referir ao financiamento, não havendo subtópicos.

Esse fato revela a falta de interesse dos pesquisadores em detalhar, organizar e divulgar suas formas de financiamento, o que dificulta a avaliação de quem lê quanto às possíveis interpretações dos vieses, dos motivos da pesquisa, dos resultados e da discussão.

Também a pouca importância dada pelos autores para aspectos técnicos da realização de sua pesquisa, dada a importância de se saber como foi o custeio de determinada pesquisa mesmo quando este foi feito com recurso próprio dos autores, a fim de facilitar possíveis reproduções futuras e testar a sua viabilidade financeira.

Torna-se importante salientar que este trabalho apresentou como limitação o fato de realizar a busca em apenas uma base de dados (PUBMED), porém não se limitou quanto ao idioma de publicação, sendo coletados os artigos disponíveis. Outra limitação até mais importante foi a especificidade do tema, associado a uma doença muito rara, o que resultou em um baixo tamanho amostral de artigos analisados. A maioria dos artigos encontrados na plataforma não fazia a associação da síndrome com a cegueira.

\section{CONSIDERAÇÕES FINAIS}

Pode-se concluir que há uma grande heterogeneidade entre os trabalhos que abordam a associação entre a SSJ e a cegueira, sendo que as principais limitações da realização desses estudos são a escassa base de dados, a raridade da doença, a demora para os casos chegarem atendimento e o longo tempo necessário para o acompanhamento. A utilização da ferramenta STROBE permitiu identificar as principais categorias com maiores índices de inadequação, sendo essas o "Viés", o "Financiamento" e o "Método". Portanto, sugere-se que os pesquisadores se atentem a melhoria das categorias com maiores inadequações, bem como, para a geração de uma base de dados referentes às complicações oculares da SSJ, fazendo parte de 
um grande banco de informações que pode resultar em um atendimento mais eficaz, objetivo e prolongado. Também, aponta-se a necessidade a realização de outros estudos, principalmente que possam definir uma relação causa-efeito entre as variáveis; e que sejam realizados de maneira padronizada entre as diferentes amostras, a fim de que possa ser feita uma comparação mais fidedigna entre estas.

\section{REFERÊNCIAS}

1. BASTUJI-GARIN S, et al. Clinical classification of cases of toxic epidermal necrolysis, Stevens-Johnson syndrome, and erythema multiforme. Archives of Dermatology, 1993; 129(1): 92-96.

2. BASU S, et al. Chronic ocular sequelae of Stevens-Johnson syndrome in children: long-term impact of appropriate therapy on natural history of disease. American Journal of Ophthalmology, 2018; 189: 17-28.

3. BRINCA A, et al. Síndrome de Stevens-Johnson e necrólise epidérmica tóxica - casuística de 10 anos. Journal of the Portuguese Society of Dermatology and Venereology, 2011; 69(3): 403-411.

4. CHRONOPOULOS A, et al. Okuläre Beteiligung bei Stevens-Johnson-Syndrom und Toxisch epidermaler Nekrolyse. Klin Monbl Augenheilkd, 2012; 229(5): 534-539.

5. DAY A, et al. The spectrum of oculocutaneous disease. American Academy of Dermatology, 2014; 70(5): 819-821.

6. ESTRELLA-ALONSO A, et al. Toxic epidermal necrolysis: a paradigm of critical illness. Revista Brasileira de Terapia Intensiva, 2017; 29(4): 499-508.

7. FRANCA M, et al. Estudo dos achados oculares na síndrome de Stevens-Johnson em pacientes de centro de referência de atendimento terciário. Arquivos Brasileiros de Oftalmologia., 2009; 72(3): 370-374.

8. GUEUDRY J, et al. Risk factors for the development of ocular complications of Stevens-Johnson syndrome and toxic epidermal necrolysis. Archives of Dermatology, 2009; 145(2): 157-162.

9. HARR T, FRENCH L. Toxic epidermal necrolysis and Stevens-Johnson syndrome. Orphanet Journal of Rare Diseases, 2010; 5(1): 39-49.

10. IYER G, et al. Treatment modalities and clinical outcomes in ocular sequelae of Stevens-Johnson syndrome over 25 years - a paradigm shift. Cornea, 2016; 35(1): 46-50.

11. JUNIOR AC, OLIVEIRA LAG. As principais causas de cegueira e baixa visão em escola para deficientes visuais. Revista Brasileira de Oftalmologia, 2016; 75(1): 26-29.

12. KOMPELLA VB, et al. Ophthalmic complications and management of Stevens-Johnson syndrome at a tertiary eye care centre in south India. Indian Journal of Ophthalmology, 2002; 50(4): 283-286.

13. MOCKENHAUPT M. The current understanding of Stevens-Johnson syndrome and toxic epidermal necrolysis. Expert Review of Clinical Immunology, 2011; 7(6): 803-815.

14. OLIVEIRA A, et al. Stevens-Johnson syndrome and toxic epidermal necrolysis. Acta Médica Portuguesa, 2011; 24(4): 995-1002.

15. OSHIKOYA K, et al. Severe cutaneous adverse drug reactions manifesting as Stevens-Johnson syndrome and toxic epidermal necrolysis reported to the national pharmacovigilance center in Nigeria: a database review from 2004 to 2017. Therapeutic Advances in Drug Safety, 2020; 11: 1-18.

16. RUIZ RB, et al. Stevens-Johnson syndrome and toxic epidermal necrolysis: a review. International Journal of Research in Medical Sciences, 2019; 7(6): 2470-2475.

17. SCHWERING MS, et al. Ocular manifestations in patients with Stevens-Johnson syndrome in Malawi-review of the literature illustrated by clinical cases. Graefe's Archive for Clinical and Experimental Ophthalmology, 2019; 257(11): 2343-2348.

18. SHANBHAG SS, et al. Long-term effect of a treatment protocol for acute ocular involvement in Stevens-Johnson syndrome/toxic epidermal necrolysis. American Journal of Ophthalmology, 2019; 208: 331-341.

19. SOLEBO A, et al. Epidemiology of blindness in children. Archives of Disease in Childhood, 2017; 102(9): $853-857$.

20. TCHETNYA X, et al. Severe eye complications from toxic epidermal necrolysis following initiation of Nevirapine based HAART regimen in a child with HIV infection: a case from Cameroon. Bmc Pediatrics, 2018; 18(108): 1-6.

21. UETA M. Results of detailed investigations into Stevens-Johnson syndrome with severe ocular complications. Investigative Ophthalmology \& Visual Science, 2018; 59(14): 183-191.

22. VAN ZYL L, et al. Prevalence of chronic ocular complications in Stevens-Johnson syndrome and toxic epidermal necrolysis. Middle East African Journal of Ophthalmology, 2014; 21(4): 332-335.

23. VANDENBROUCKE JP, et al. Strengthening the Reporting of Observational Studies in Epidemiology (STROBE): Explanation and elaboration. International Journal of Surgery, 2014; 12(12): 1500-1524.

24. WONG A, et al. Stevens-Johnson syndrome and toxic epidermal necrolysis: a review. Revista da Associação Médica Brasileira, 2016; 62(5): 468-473.

25. YAMANE Y, et al. Analysis of Stevens-Johnson syndrome and toxic epidermal necrolysis in Japan from 20002006. Allergology International, 2007; 56(4): 419-425. 